of working time. Moreover, 'High physical demanding service' were significantly concerned by largest fraction of handling time $(1.77 \%)$, by lower degree of autonomy of handled patients $(1.51 \%$ of total working time), and by handling patients having weight exceeding $65 \mathrm{~kg}(1.57 \%$ of total working time). According to the Chamoux scale, global physical activity was classified rather heavy in all services groups, with an average score significantly increasing from 7.25 to 7.76 from' light' to 'high' physical demanding services $($ Anova $=0.039)$.

Conclusion Our investigation objectified variable levels of biomechanical constraints in hospital services. Ergonomic and organizational preventing measures should be set up to prevent serious consequences among caregivers, in particular those affected to heavy services.

\section{P-260 FATAL WORK-RELATED INJURIES INVOLVING MOTORCYCLE BY SEX, AGE AND OCCUPATIONAL SECTORS IN BRAZIL, 2007 TO 2018}

${ }^{1}$ Cleber Cremonese, Homègnon Antonin Ferréol Bah, Mateus Lisboa, Aline Cristina Gusmão, Lenira Ferreira Ribeiro, Rafael Buralli, Flávia Ferreira-Sousa, Eugenia Maria Rodrigues, Vilma Sousa Santana. 'Federal University of Bahia, Brazil

\subsection{6/OEM-2021-EPI.256}

Introduction Brazilian records show a high rate of traffic accidents involving motorcycles (30\% of deaths, 55\% of hospitalizations). The number of Brazilian workers who use motorcycles is increasing and it is necessary to understand the context of these accidents in order to develop public policies and promote education.

Objectives To describe the proportion of mortality by workrelated injuries (PM_WRI) involving motorcycle in Brazil, 2007-2018.

Methods The study was conducted with data from the Brazilian Mortality Information System for those aged 18-65 years, in 2007-2018. Motorcycle-related deaths correspond codes V20 to V29 (ICD-10). PM_WRI are presented by calendar year, sex, age and occupational groups.

Results There were 121,124 records of fatal injuries involving motorcycles, with a increasing linear trend from 7,502 in 2007 to 9,725 in 2018. Work-related data (WRD) were registered for only 48,716 (40.2\%) cases, from which 3,692 were classified as occupational. From 2007 to 2010, the PM_WRI went from $7.6 \%$ to $8.9 \%$ (maximum) when it started to fall until 2015 (6.4\% minimum). The average for the last three years was $7.4 \%$. No significant difference of PM_WRI by sex was found. PM_WRI increased with age but declined in the oldest age group (50-65 age years). Occupation was registered for 33,784 cases (69\% of the WRD). The highest PM_WRI was estimated among workers from service industry $(1 \overline{4} .3 \%)$ followed by administrative services (13.6\%). Agriculture had the largest number of motorcycle-related deaths (35\%) but only $3.1 \%$ was recognized as work-related.

Conclusion The work-related data in death certificates were poorly recorded, limiting conclusions on the contribution of labor on motorcycle associated deaths. PM WRI estimates were presumably underestimated and findings could be biased. Motorcycle-related deaths doubled over the study time and the role of labor for this need to be better understood. Improvements in the quality and completion of WRD are urgently needed and prevention programs implemented.

\section{P-261 SEMI-QUANTITATIVE ERGONOMIC ASSESSMENT OF BIOMECHANICAL RISK FACTORS OF THE UPPER LIMBS' MUSCULOSKELETAL DISORDERS AMONG TUNISIAN JEWELRY CRAFTSMEN}

'Lamia Bouzgarrou, Ben Afia Latifa, Asma Kheder, Amira Omrane, Faten Hermassi, Taoufik Khalfallah. 'University of Monastir, Tunisia

\subsection{6/OEM-2021-EPI.257}

Introduction Jewelry handcrafting activity is strongly associated with biomechanical constraints and high risk of upper limb musculoskeletal disorders (UL-MSDs).

Objective This study aims to assess biomechanical constraints and specific tasks and subtasks at risk of UL-MSDs among jewelry craftsmen.

Methods Open observations were conducted in several jewelry workshops during whole working days. These observations allowed us to identify representative work periods, tasks and subtasks and to set the number of recordings at fourteen according to 'homogeneous exposure group' (HEG) sampling recommendations. For each video recording (duration $=30$ to 40 minutes), 100 images were extracted through regular stops on fixed time intervals. In each image, postures were encoded and analyzed by ERGOROM software. Gestural Variability Score (GSV) was calculated based on variability of posture from one image to the next.

Results Six main tasks composed handcrafting jewelry activity (mold making; fusion and gluing metal; demoulding; sanding; crimping and polishing). These tasks were associated to eleven subtasks (the creation of impression in the mold, the heating of the metal, the welding during assembly of the piece.). Objective analysis revealed that jewelers spent 38\%of their working time with the neck in flexion exceeding $40^{\circ}$ or extension, and the shoulders in adduction, extension or visible rotation during $60.8 \%$ of the time. The elbow articulation was maintained in extreme pronation in $40.4 \%$ of the working time and flexion between $60^{\circ}$ and $100^{\circ}$ during $78.1 \%$ this time. The ulnar deviation of the wrist was observed during $40.9 \%$ of work time and the pinch grip for over $79.5 \%$ of it. In addition, the high variability score was noted especially for elbow joint and shoulder, reflecting a rapid postural change and high repetitiveness.

Conclusion Our results, suggest the necessity of preventive actions among jewelers craftsmen, with mainly the introduction of ergonomic design of hand tools .

\section{P-268 METAL EXPOSURE AND RISK OF PARKINSON'S DISEASE: SYSTEMATIC REVIEW AND META-ANALYSES}

${ }^{1}$ Yujia Zhao, Susan Peters, Roel Vermeulen, Anushree Ray. 'Utrecht University, Netherlands

\subsection{6/OEM-2021-EPI.258}

Introduction Parkinson's disease (PD) is the second most common neurodegenerative disorder. Metal exposure has been suggested as a possible environmental risk factor by many epidemiological studies, but results have been inconsistent. Additionally, existing reviews on metal exposure and PD risk lack careful screening for study design and quality, especially on the exposure assessment.

Objectives We aimed to synthesize the literature on metal exposure and PD risk by examining the quality of the overall study and exposure assessment method. 
Methods We conducted a systematic review on observational studies of metal levels from biological matrices, and dietary and occupational/environmental sources among PD patients and controls. We searched the PubMed/MEDLINE, EMBASE and Cochrane databases up to July 2020. Metal species included manganese, iron, copper, lead, mercury, aluminum, calcium, selenium, zinc, magnesium, cadmium, chromium and nickel, and the outcome was idiopathic PD. We applied an adapted Newcastle-Ottawa Scale (NOS) and a previously established exposure assessment rating to evaluate each individual study. We then performed meta-analyses with random-effects model.

Results 80 case-control studies were included, of which 69 were graded as low or moderate quality. The majority of casecontrol studies were hospital-based and applied biomonitoring approaches to quantify metal levels after disease diagnosis. Studies on copper, iron, manganese and zinc were most prevalent. Meta-analyses showed no significant PD risk for these metals and heterogeneity among studies was substantial. Furthermore, 5 cohort studies were retained, but the population source, metal exposure and follow-up period were heterogeneous.

Conclusion The level of evidence on metal exposure and PD risk is limited and no consensus can be drawn from the literature. Reverse causality cannot be ruled out by existing biomonitoring studies. Studies assessing metal levels before disease onset are needed to improve our understanding of the role of metals in the etiology of PD.

\section{P-279 RETURN TO WORK AFTER PARENTAL LEAVE: PERSPECTIVE OF BRAZILIAN WOMEN}

${ }^{1}$ Leticia Santos, Vivian Mininel. 'Universidade Federal de São Carlos, Brazil

\subsection{6/OEM-2021-EPI.259}

Introduction maternity leave is a constitutional right in Brazil, but the payment/benefits is restricted to women who contribute to the social security system. Although women represent half of the labor workforce around the world, they are still mostly responsible for family and child-rearing which compromises the process of return to work after birth.

Objective to understand the return to work after maternity leave from the perspectives of Brazilian working women.

Methods qualitative study with mothers that gave birth and return to work regardless of kind of job. Until now, four women have participated in online interviews following a validated script of open questions. Data analysis has been done simultaneously of data collected through thematic approach and the sample will be delimited by saturation. This research was approved by the Research Ethics Committee of the Federal University of Sao Carlos, Brazil (all ethical concerns have been respected).

Results return to work influenced the continuation of breastfeeding, including home office: 'I was in front of the computer and breastfeeding'. There is a duality when women talk about job and the care with the babies: 'the good thing is that I manage to stay with him all the time [...] and at the same time it also becomes exhausting to stay at home for a long time'. Women report that the work was invaded by the needs of their children causing dissatisfaction and frustration with performance after returning: 'I feel more encouraged to work [...] but my career is over, at least in the pandemic there is no way', especially during pandemic with all day care centers closed.

Conclusion the burden of responsibility in caring for children falls on women impacting performance at work and also breastfeeding.

\section{P-280 METALWORKING FLUIDS AND CANCER INCIDENCE IN THE UAW-GM AUTOWORKERS COHORT}

${ }^{1}$ Hilary Colbeth, Kevin Chen, Sally Picciotto, Sadie Costello, Ellen Eisen. ${ }^{1}$ University of California Berkeley, United States

\subsection{6/OEM-2021-EPI.260}

Introduction Metalworking fluids (MWF) are complex mixtures of oils and chemical additives used to cool and lubricate metal machining operations. Previous studies have reported increased risk of specific cancers associated with MWF exposure.

Objectives This report broadly examines cancer incidence in the United Auto Workers-General Motors (UAW-GM) cohort exposed to MWFs with extended follow-up (through 2015). The outcomes of interest were melanoma, leukemia, nonHodgkin lymphoma and cancers of the colon, rectum, pancreas, esophagus, stomach, larynx, lung and bronchus, breast, prostate, kidney and renal pelvis, and bladder.

Methods The cohort includes 39,132 workers followed for cancer incidence in Detroit area Surveillance, Epidemiology, and End Results Program (SEER) and Michigan cancer registries, from 1973-2015. Cox models yielded estimates of adjusted hazard ratios (HR) with categorical variables for lagged cumulative exposure to each MWF (straight, soluble, and synthetic).

Results There were 7,809 cancer cases of interest. Over 43 years of follow-up, the incidence of several types of cancers was significantly elevated in relation to at least one type of MWF; exposure-response patterns were consistent with prior reports from this cohort. We found significantly increased incidence of stomach and kidney cancer associated with higher levels of straight fluid exposure and increased rectal and pancreatic cancer with increasing synthetic fluid exposure. Only Non-Hodgkin lymphoma was associated with soluble MWF, with HRs significantly elevated in the highest exposure category at 1.70 (95\% Confidence Interval (CI): 1.13-2.54).

Conclusions Our results provide further evidence of associations between MWF exposure and several types of cancer. This study summarizes information on the incidence of the fourteen cancer types with reduced bias from both the healthy worker hire effect and left truncation. However, the HRs presented do not address potential downward bias from the healthy worker survivor effect which may be necessary to correct in future targeted analyses.

\section{P-281 IDENTIFYING RETURN-TO-WORK TRAJECTORIES AMONG BREAST CANCER SURVIVORS USING SEQUENCE ANALYSIS}

${ }^{1}$ Mélanie Bertin, Yves Roquelaure, Elise Rubion, Bertrand Porro, Fabien Gilbert. 'École des hautes études en santé publique (EHESP), France

10.1136/OEM-2021-EPI.261 